\title{
Association of early elevated cardiac troponin I concentration and longitudinal change after off-pump coronary artery bypass grafting and adverse events: a prospective cohort study
}

\author{
Yan Li, Yuqi Li, Qiuming Hu, Shuai Zheng, Baiyu Tian, Fei Meng, Zonghao Chen, Jie Han, \\ Shengyu Wang, Haibo Zhang, Chunlei Xu, Yixin Jia, Yuqing Jiao, Jintao Fu, Xu Meng \\ Beijing Anzhen Hospital, Capital Medical University, Beijing, China \\ Contributions: (I) Conception and design: Y Li, X Meng, J Fu; (II) Administrative support: Y Li, X Meng; (III) Provision of study materials or patients: \\ Y Li, Y Li, J Fu; (IV) Collection and assembly of data: Y Li, Y Li, Q Hu, B Tian, F Meng, Z Chen, J Han, S Wang, H Zhang, C Xu, Y Jia, Y Jiao, \\ J Fu, X Meng; (V) Data analysis and interpretation: Y Li, Y Li, J Fu; (VI) Manuscript writing: All authors; (VII) Final approval of manuscript: All \\ authors. \\ Correspondence to: Xu Meng. Beijing Anzhen Hospital, Capital Medical University, No. 2 Anzhen Road, Chaoyang District, Beijing 100029, China. \\ Email: mengxu191116@163.com.
}

\begin{abstract}
Background: The elevation of troponin after coronary artery bypass grafting (CABG) is common This study aimed to investigate the association between very early cardiac troponin I (cTnI) concentration and its longitudinal change within 24 hours after CABG and 30-day adverse events.

Methods: This study prospectively enrolled 633 patients who underwent isolated off-pump CABG from January 2019 to May 2019. Serum cTnI levels were measured in all patients at two examinations within 24 hours postoperatively (1 hour and 12-18 hours), and a proportional hazards model was used to determine the association between cTnI levels and their change with adverse events, which were defined as a composite of 30-day mortality, stroke, heart failure, myocardial infarction (MI), and ventricular fibrillation.

Results: cTnI levels of the two examinations and absolute change of c $T n I$ levels were significantly higher in the event group than in the non-event group $(\mathrm{P}<0.01$, both). Earlier and later $\mathrm{c} T \mathrm{Tn}$ concentrations were associated with 30-day complications [adjusted hazard ration (HR) 1.598, 95\% confidence interval (CI), 1.158-2.204 and HR 1.499, 95\% CI, 1.228-1.831, respectively]. With regard to longitudinal change in cTnI levels, participants with persistently high levels of cTnI and those with progression from a low level to high level concentration experienced a significantly increased risk of adverse events than did participants who had a trend of persistently low c'TnI levels (HR 3.105, 95\% CI, 1.748-5.517 versus HR 2.944, 95\% CI, 1.488-5.824).

Conclusions: Longitudinal change in cTnI levels within 24 hours and early cTnI concentrations, even less than 1 hour after CABG, are associated with adverse events. These data will be useful in identifying patients at an increased risk of complications.
\end{abstract}

Keywords: Coronary artery bypass grafting (CABG); cardiac troponin I (cTnI); longitudinal change; prognosis

Submitted Apr 16, 2020. Accepted for publication Sep 17, 2020.

doi: 10.21037/jtd-20-1691

View this article at: http://dx.doi.org/10.21037/jtd-20-1691

\section{Introduction}

Coronary artery bypass grafting (CABG) remains the most common cardiac surgical procedure and effective revascularization strategy for coronary artery disease (CAD) with outstanding long-term clinical outcomes, particularly for multivessel and left main disease (1). However, severe perioperative myocardial injury or cardiac dysfunction, which may be caused by ischemia-reperfusion injury, acute graft failure, and operative trauma, is associated with increased incidence of adverse events (2). Early detection of 
complications after CABG is important to perform timely therapeutic strategies, such as more aggressive medication, urgent re-exploration of the graft with or without redo revascularization (CABG or percutaneous intervention), and mechanical circulation assist (intra-aortic balloon pump), to reduce the risk of morbidity. Cardiac troponin is the most frequently used biomarker for detecting myocardial injury and cardiac dysfunction after CABG, and elevation of troponin levels within the first 24 hours is independently associated with worse outcomes $(3,4)$. Several studies have been performed to investigate the association between postoperatively elevated troponin concentration and the risk of complications; nevertheless, there were some limitations. (I) A previous study assessed the troponin level on the first postoperative day (5), whereas some other studies advanced the time to less than 24 hours $(3,6)$ or even 6-12 hours postoperatively (7); thus, the value of troponin less than 6 hours postoperatively is unclear. (II) Previous studies tended to evaluate troponin at a fixed time point; hence, data regarding the longitudinal change of troponin during the period within the first 24 hours after CABG are scarce. (III) CABG with or without cardiopulmonary bypass $(\mathrm{CPB})$ and $\mathrm{CPB}$ time have significantly affected troponin levels (8). The current study specifically focused on offpump CABG, whereas most former studies were performed among patients who underwent on-pump CABG. Because of the aforementioned limitations $(7,9)$, we hypothesized that there would be a significant association between very early elevated cardiac troponin I (cTnI) concentration and its longitudinal change after off-pump CABG and adverse events. Therefore, this study aimed to investigate the association between very early $\mathrm{c} T \mathrm{Tn}$ concentration and its longitudinal change within 24 hours after CABG and 30day adverse events. We present the following article in accordance with the STARD reporting checklist (available at http://dx.doi.org/10.21037/jtd-20-1691).

\section{Methods}

\section{Study design and patients}

This prospective study enrolled consecutive patients with CAD who underwent CABG from January 2019 through May 2019 at a cardiac center, Beijing Anzhen Hospital, Beijing, China. All patients received CABG in accordance with contemporary practice guidelines (1), heart team judgments, and patient preferences. Patients who underwent other concomitant cardiovascular surgery were excluded, while patients who underwent off-pump CABG and had at least two cTnI examinations within the first 24 hours in the intensive care unit (ICU) after surgery were included in this study. There were no other prespecified exclusion criteria. Comprehensive clinical data (demographics, disease history, baseline echocardiographic data, and laboratory test results) were collected for each subject. The study was conducted in accordance with the Declaration of Helsinki (as revised in 2013). The study was approved by institutional ethics board of Beijing Anzhen Hospital (No. ks2019018). All participants provided informed consent before taking part.

\section{Determination of $c$ TnI and its longitudinal change}

Blood samples were taken from patients by direct venipuncture when they returned to the ICU for determination of the cTnI level. Every patient had at least had two serum cTnI results at different time points. The first postoperative blood sample for testing $\mathrm{c} T \mathrm{Tn}$ (named cTnI-1) was drawn immediately after patients arrived at the surgical ICU (less than 1 hour after CABG), and the second blood sample (named cTnI-2) was drawn 12 to 18 hours after the procedure. The longitudinal change of cTnI within the first 24 hours was calculated as follows: cTnI-1 concentration - cTnI-2 concentration. The normal range for serum c $\mathrm{TnI}$ in our laboratory was 0.01 to $0.023 \mu \mathrm{g} / \mathrm{L}$.

\section{Outcomes}

Outcome data were acquired by in-hospital record review, clinic visit, and telephone interview. Adverse events were independently confirmed by two authors in order to reduce bias. The primary endpoint of the study was the rate of a composite of 30-day all-cause mortality, stroke, myocardial infarction (MI), heart failure, and ventricular fibrillation after surgery. Outcome was defined in accordance with the Guidelines on myocardial revascularization (1).

\section{Statistical analysis}

Continuous data that were abnormally distributed are expressed as median [interquartile range (IQR)], and categorical data are presented as number (percentage). Change in cTnI was modelled as both categorical and continuous variables, and it is expressed as a categorical variable using quartiles and trends. Change expressed in trends was divided into four groups according to cTnI concentrations at the first and second sampling times 
relative to the median of each time: high to high, high cTnI-1 and high cTnI-2 concentrations; high to low, high cTnI-1 and low cTnI-2 concentrations; low to high, low cTnI-1 and high cTnI-2 concentrations; and low to low, low cTnI-1 and low cTnI-2 concentrations. Differences of baseline characteristics were compared using the MannWhitney $\mathrm{U}$ test or Kruskal-Wallis $\mathrm{H}$ test for continuous variables and the Pearson $\chi^{2}$ test or Fisher exact test for categorical variables. To examine the effect of the cTnI level at different time points and on clinical outcomes, proportional Cox hazard models were used to calculate the hazard ratio (HR). To analyze cTnI-1, cTnI-2, and absolute change, the models were adjusted for hypertension, left ventricular end-diastolic diameter (LVEDD), and left ventricular ejection fraction (LVEF), which were statistically different between the no event and event groups. Regarding longitudinal change, we adjusted LVEF for quartiles and sex, hemoglobin level, and platelet counts for trends, respectively. The receiver operating characteristic curve and area under the curve (AUC) were used to assess the ability of $\mathrm{cTnI}$ concentrations at different time points and their longitudinal change to predict 30 -day adverse events. A two-sided $\mathrm{P}$ value $<0.05$ indicated a significant difference. All analyses were performed using Stata version 15.2 for Windows (StataCorp, College Station, TX, USA).

\section{Results}

A total of 633 participants who underwent only off-pump CABG procedure (median age 63 years, range, 35-87 years, $74 \%$ men) with available early postoperative cTnI data were included in our study. The median EUROSCORE II was $1.01 \%$ (IQR $0.71-1.775 \%$ ). The median cTnI concentrations were markedly elevated at first and second sampling times $(0.140 \mu \mathrm{g} / \mathrm{L}$, IQR $0.067-0.260 \mu \mathrm{g} / \mathrm{L}$ and $0.130 \mu \mathrm{g} / \mathrm{L}$, IQR $0.063-0.340 \mu \mathrm{g} / \mathrm{L}$, respectively). cTnI levels changed within 24 hours after the procedure. Table 1 shows the baseline characteristics of the study participants by two division methods: longitudinal change of cTnI expressed in quartiles and trends. A significant difference was observed in baseline LVEF across the four quartiles $(\mathrm{P}=0.031)$. When examining two cTnI levels relative to the median of each sampling time, we found that $15.2 \%$ of the patients changed from low to high concentrations, and $13.3 \%$ of the patients changed from high to low $\mathrm{c} T n \mathrm{I}$ concentrations. By contrast, about $33.6 \%$ of the patients had persistently low c TnI concentrations, and $37.9 \%$ had persistently high cTnI levels. There were statistically significant differences in the proportion of men and preoperative hemoglobin and platelet levels among the four groups $(\mathrm{P}<0.05$, all). In total, 101 patients $(16.0 \%)$ experienced adverse events within 30 days postoperatively, comprising seven deaths, 13 patients with MI, 70 with heart failure, eight with ventricular fibrillation, and 13 with stroke (several patients sustained two or more complications).

Participants who developed complications had larger LVEDD and lower LVEF, and they were more likely to have hypertension $(\mathrm{P}<0.05$, all). Overall, 484 patients $(76.5 \%)$ received three or more grafts for CABG.

Higher $\mathrm{c} T \mathrm{Tn}$ levels at the first and second sampling times after CABG were observed in the event group than in the no event group $(\mathrm{P}=0.002$ and $<0.001$, respectively). Absolute change in $c T n I$ levels which were defined as absolute value of first time cTnI concentration minus second time cTnI concentration was higher in the event group than in the no event group $(\mathrm{P}<0.001$, Figure 1 and Table 2).

Both continuous cTnI-1 and dichotomized cTnI-1 levels at the cutoff of $0.065 \mu \mathrm{g} / \mathrm{L}$, which was identified by the maximum Youden index value, were significantly associated with 30-day adverse events (adjusted HR for the continuous cTnI-1 level 1.598, 95\% CI, 1.158-2.204; adjusted HR for the dichotomous cTnI-1 level 3.275, 95\% CI, 1.613-6.651; Table 3). Significant associations were also observed between cTnI-2 and 30-day adverse events (adjusted HR for the continuous cTnI-2 level 1.499, 95\% CI, 1.228-1.831; adjusted HR for the dichotomous cTnI-2 level 3.541, 95\% CI, 2.177-5.759). Absolute change in cTnI was associated with a $38 \%$ increase in the risk of complications (adjusted HR for continuous absolute change 1.381, 95\% CI, 1.1381.674).

When longitudinal change in cTnI concentrations was $\geq 0.143 \mu \mathrm{g} / \mathrm{L}$ regardless of whether the trend was up or down, patients experienced a $142 \%$ increase in the risk of adverse events (adjusted HR for the dichotomous absolute change $2.427,95 \%$ CI, $1.527-3.856$, Table 3 ). The ability of cTnI-2 (AUC: 0.682) for predicting 30-day adverse events after CABG was relatively higher than the abilities of cTnI-1 and absolute change (AUC: 0.596 and 0.646, respectively; Figure 2).

Longitudinal change in cTnI within 24 hours after CABG was expressed in quartiles and trends. When presented in quartiles, we found that patients in quartile $1(14.6 \%)$ and quartile $4(28.5 \%)$ had a higher proportion of incident adverse events than those in quartile $2(10.4 \%)$ and quartile $3(10.4 \%)$. When expressed in trends, we found higher incidences of adverse events in the low to high 


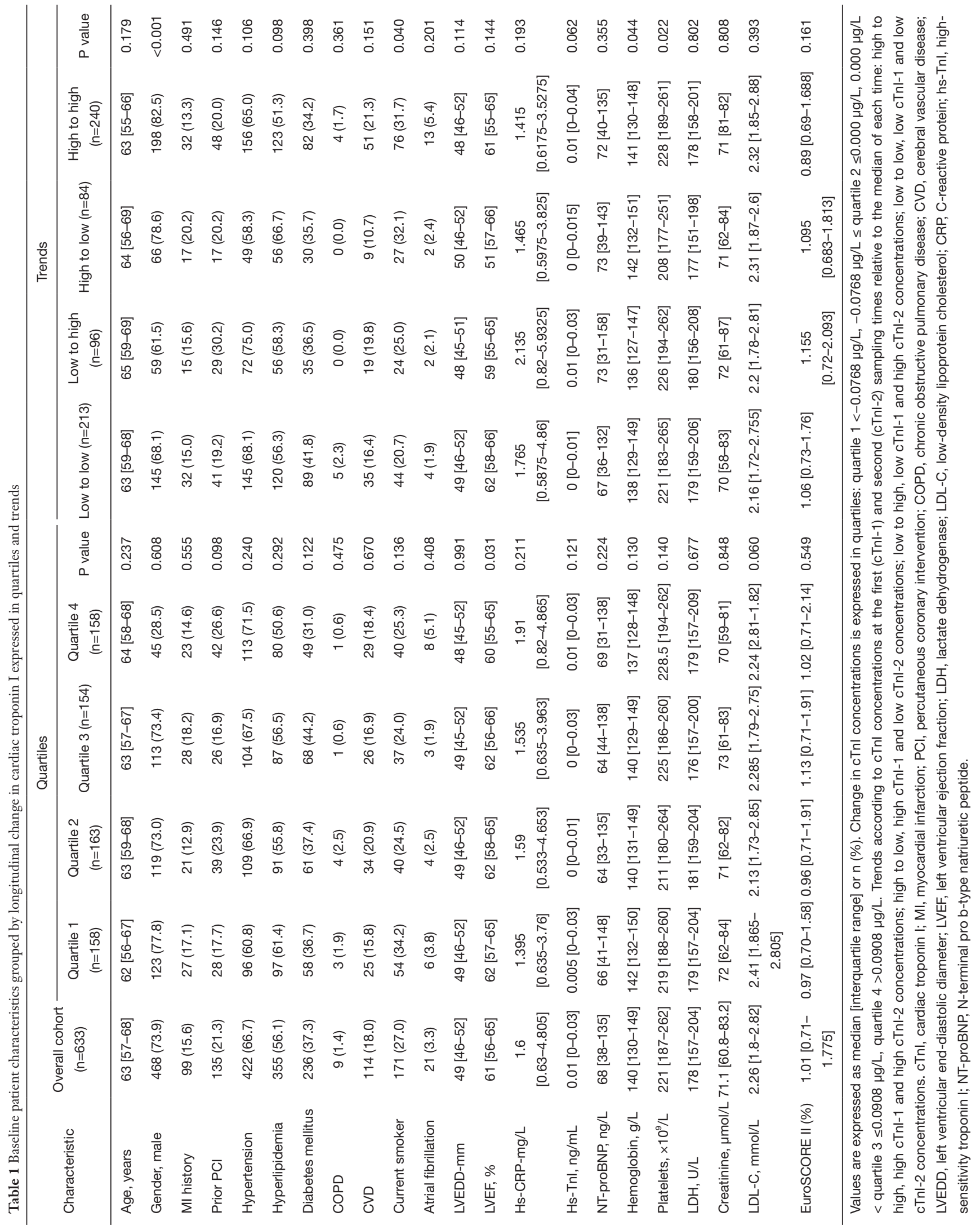


(24.0\%) and high to high $(21.3 \%)$ groups than in the low to low $(8.9 \%)$ and high to low $(9.5 \%)$ groups (Table 2 and Figure 3). Patients in quartile 4 experienced a 2.34 times higher risk of adverse events than those in quartile 1 (HR 2.337, 95\% CI, 1.334-4.097, $\mathrm{P}=0.003$ ).

Further, this significant association remained even after adjustment for baseline LVEF (HR 2.419, 95\% CI, 1.319-4.438, $\mathrm{P}=0.004$; Figure 4). Patients with progression

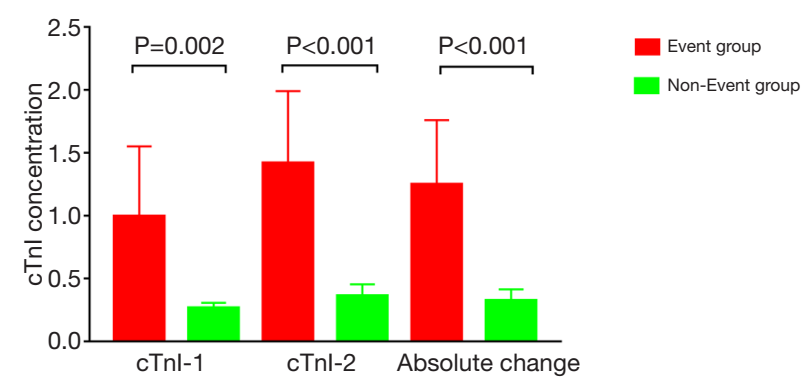

Figure 1 Difference in the cardiac troponin I level between the event group and no event group. cTnI, cardiac troponin I; cTnI1 , measurement of cardiac troponin I levels less than 1 hour after CABG; cTnI-2, measurement of cardiac troponin I levels between 12-18 hours after coronary artery bypass grafting. from low levels at the earlier time to high levels at the later time (low to high) showed a 2.94-fold higher incidence of 30 -days adverse events after CABG than those with low to low levels (adjusted HR 2.944, 95\% CI, 1.488-5.824, $\mathrm{P}=0.002)$. Moreover, patients whose $\mathrm{c} T \mathrm{TnI}$ concentrations changed from high to low had an adjusted HR of 1.227 (95\% CI, 0.511-2.945, $\mathrm{P}=0.646$ ). Those with persistently high cTnI levels had a 2.76 times higher risk of postoperative complications than those without (HR 2.755, 95\% CI, 1.568-4.841, $\mathrm{P}<0.001)$; moreover, the association became more significant after adjusting for male sex and preoperative hemoglobin and platelet levels (adjusted HR 3.105, 95\% CI, 1.748-5.517, P<0.001; Figure 4).

\section{Discussion}

The main findings of our study are as follows. First, we found that the $\mathrm{c} T \mathrm{nI}$ concentration at a fixed time point within 24 hours after off-pump CABG is significantly associated with 30-day postoperative composite adverse events of all-cause mortality, heart failure, MI, stroke, and ventricular fibrillation. The significant association between cTnI levels within 1 hour after surgery and adverse events can provide a potential reference for more aggressive

Table 2 Difference of the troponin I level between the event and no event groups and the proportion of events

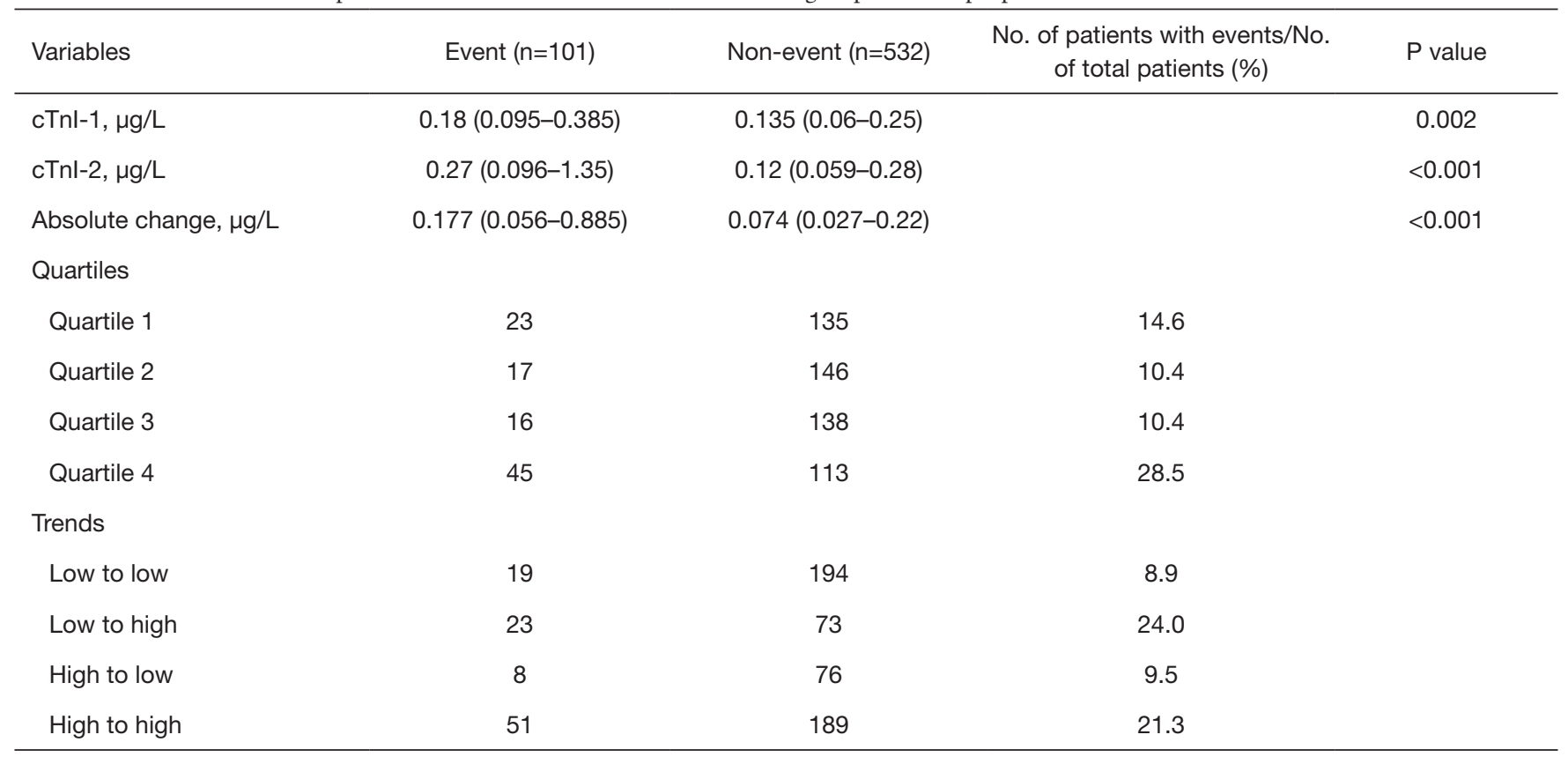

Values are expressed as median (interquartile range) or $\mathrm{n} . \mathrm{cTnl}-1$ = measurement of cardiac troponin I levels less than 1 hour after CABG; cTnl-2 = measurement of cardiac troponin I levels between 12-18 hours after coronary artery bypass grafting; absolute change in cTnl levels $=$ absolute value of the first sampling time of the cTnl concentration minus the second sampling time of the cTnl concentration. 
Table 3 Association between cardiac troponin I and incident outcomes

\begin{tabular}{lcccc}
\hline Item & No. of patients & $\begin{array}{c}\text { No. of patients with } \\
\text { events }\end{array}$ & Unadjusted HR (95\% Cl) & Adjusted HR (95\% Cl) \\
\hline cTnl-1 & 633 & 101 & $1.62(1.237-2.122)$ & $1.598(1.158-2.204)$ \\
$\geq 0.065$ (cutoff) & 154 & 89 & $2.7(1.434-5.084)$ & $3.275(1.613-6.651)$ \\
$<0.065$ & 479 & 12 & Reference & - \\
cTnl-2 & 633 & 101 & $1.483(1.252-1.757)$ & $1.499(1.228-1.831)$ \\
$\geq 0.395$ (cutoff) & 141 & 46 & $3.847(2.453-6.034)$ & $3.541(2.177-5.759)$ \\
$<0.395$ & 492 & 55 & Reference & - \\
Absolute change & 633 & 101 & $1.378(1.191-1.595)$ & $1.381(1.138-1.674)$ \\
$\geq 0.143$ (cutoff) & 224 & 55 & $2.568(1.667-3.956)$ & 2.427 (1.527-3.856) \\
$<0.143$ & 409 & 46 & Reference & - \\
\hline
\end{tabular}

The cutoff value was identified by the maximum Youden index value. HR, hazard ratio; Cl, confidence interval; cTnl-1, measurement of cardiac troponin I levels less than 1 hour after CABG; cTnl-2, measurement of cardiac troponin I levels between 12-18 hours after coronary artery bypass grafting.

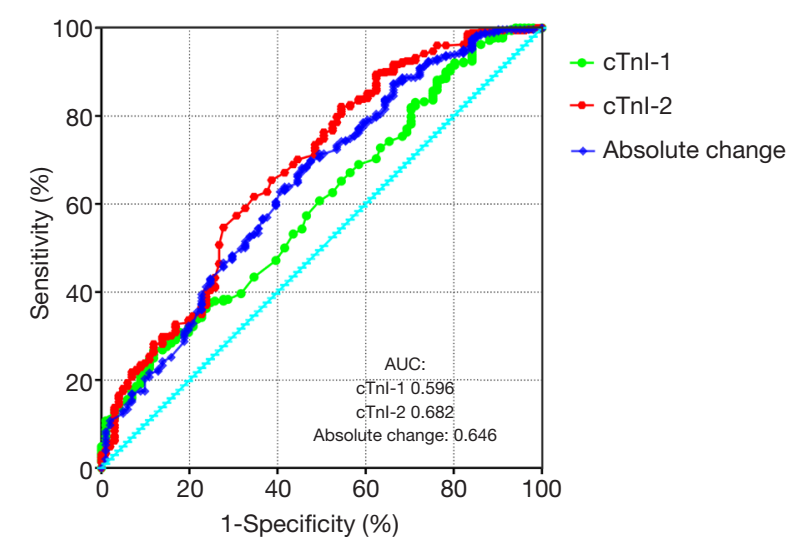

Figure 2 Receiver operative characteristic curve for predicting adverse events. cTnI-1, measurement of cardiac troponin I levels less than 1 hour after CABG; cTnI-2, measurement of cardiac troponin I levels between 12-18 hours after coronary artery bypass grafting; AUC, area under the curve.

treatment to reduce the subsequent risk of complications. Second, we demonstrated that longitudinal change in the $\mathrm{c} T \mathrm{nI}$ concentration within 24 hours postoperatively is independently associated with the development of 30-day composite adverse events. Specifically, patients with persistently high cTnI concentrations and those with progression from low to high cTnI concentrations had a more than three times increased risk of adverse events than those who had persistently low cTnI concentrations. Third, cTnI levels at a fixed time point and absolute change

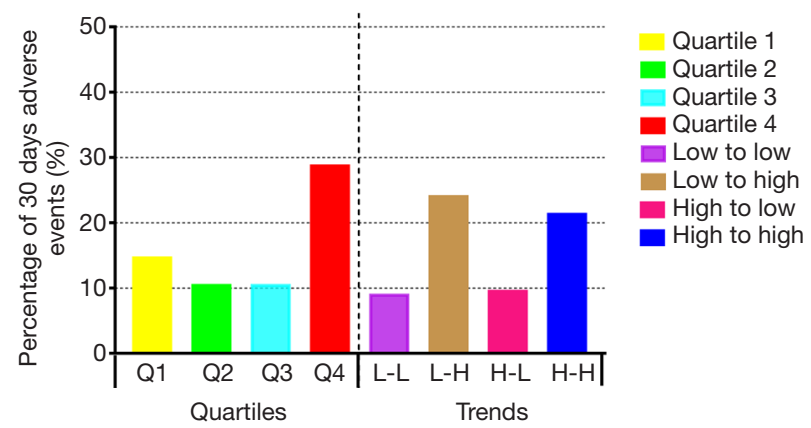

Figure 3 Proportion of adverse events in each longitudinal change group. Longitudinal change in cardiac troponin I levels within first 24 hours after coronary artery bypass grafting is expressed in quartiles and trends. L, low; H, high.

within 24 hours after CABG have some value for predicting adverse events (Figure 5).

CABG is among the most frequently performed cardiac surgical procedures $(10,11)$, giving public concern to factors that adversely affect the outcomes of this procedure. The perioperative c $\mathrm{TnI}$ concentration is suggested to be one of the most sensitive and accurate myocardial biomarkers for identifying MI and predicting clinical outcomes following cardiovascular surgery $(1,12,13)$; it even be used to discern between patients with graft-related MI, those with nongraft-related MI, and those without perioperative MI after CABG (14). A previous study reported that preoperative troponin measurement ahead of emergency CABG was 
A

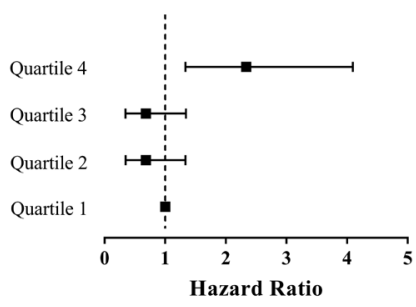

C

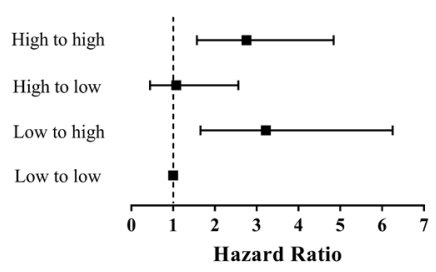

B
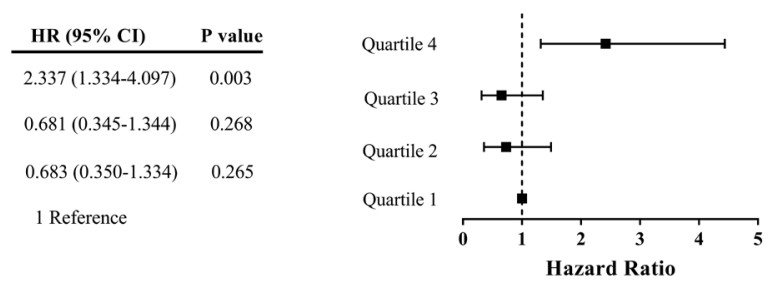

Adjusted HR(95\%CI) P value

$2.419(1.319-4.438) \quad 0.004$

$0.654(0.315-1.355) \quad 0.253$

$0.732(0.358-1.494) \quad 0.391$

1 Reference

D

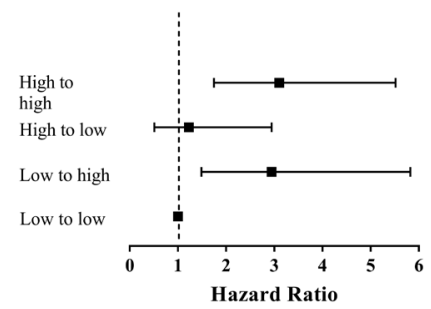

Adjusted HR(95\%CI) P value

$3.105(1.748-5.517)<0.001$

$1.227(0.511-2.945) \quad 0.646$

2.944(1.488-5.824) $\quad 0.002$

1 Reference

Figure 4 Association between longitudinal change in cardiac troponin I levels and adverse events. Change in cTnI concentrations is expressed in quartiles: quartile $1<-0.0768 \mu \mathrm{g} / \mathrm{L},-0.0768 \mu \mathrm{g} / \mathrm{L} \leq$ quartile $2 \leq 0.000 \mu \mathrm{g} / \mathrm{L}, 0.000 \mu \mathrm{g} / \mathrm{L}<$ quartile $3 \leq 0.0908 \mu \mathrm{g} / \mathrm{L}$, quartile $4>0.0908 \mu \mathrm{g} / \mathrm{L}$. Change in cTnI concentrations is expressed in trends according to cTnI concentrations at the first and second sampling times relative to the median of each time: high to high, high cTnI-1 and high cTnI-2 concentrations; high to low, high cTnI-1 and low cTnI-2 concentrations; low to high, low c'TnI-1 and high cTnI-2 concentrations; low to low, low cTnI-1 and low c'TnI-2 concentrations. cTnI-1, measurement of cardiac troponin I levels less than 1 hour after CABG; cTnI-2, measurement of cardiac troponin I levels between 12-18 hours after coronary artery bypass grafting; cTnI-1, cardiac troponin I.

an independent and powerful determinant of all-cause inhospital mortality and major adverse cardiac events in acute ST-elevation MI and non-ST-elevation MI (15). However, other investigations suggested that there was no association between preoperative troponin and 30-day major adverse events in an elective CABG population or mixed population that included elective and emergency surgical patients $(5,16)$, and even in the setting of general cardiac surgery, the preoperative troponin level was not associated with postoperative long-term mortality (17). Therefore, measurement of early postoperative troponin levels becomes necessary and meaningful, and it is not surprising that troponin levels are commonly elevated after CABG, as this elevation may result from insufficient myocardial protection during surgery, air embolism, graft occlusion, or other reasons $(3,18-20)$.

Although the troponin concentration after CABG is affected by many factors (8), for instance, preoperative MI, kidney function, and LVEF, previous studies reported the promising ability of early troponin levels to predict clinical outcomes following the procedure. A large-scale prospective longitudinal study demonstrated that troponin was superior to electrocardiogram findings and creatinine kinase- $M B$ with regard to predicting 5-year mortality after CABG (21); furthermore, the predictive value of troponin for 5-year mortality was validated in a separately collected cohort of 1,031 individuals who underwent CABG. In addition, another high-quality meta-analysis which included 18,908 patients (3), suggested that elevation of troponin levels within the first 24 hours after CABG was independently associated with increased mid- and long-term incidences of mortality. Nevertheless, the reality, which is easy to neglect, is that many adverse events occur immediately after CABG and urgent therapeutic measures are needed to improve the prognosis of these patients. Because the effective treatment time window narrows quickly, it is necessary to advance the time of measuring postoperative troponin levels. Gahl and colleague demonstrated that troponin levels measured between 6 and 12 hours after CABG can be used to identify individuals at an increased risk of major adverse events (7). Very few studies focused on the prognostic use of an early assessment of troponin within 6 hours after CABG to 

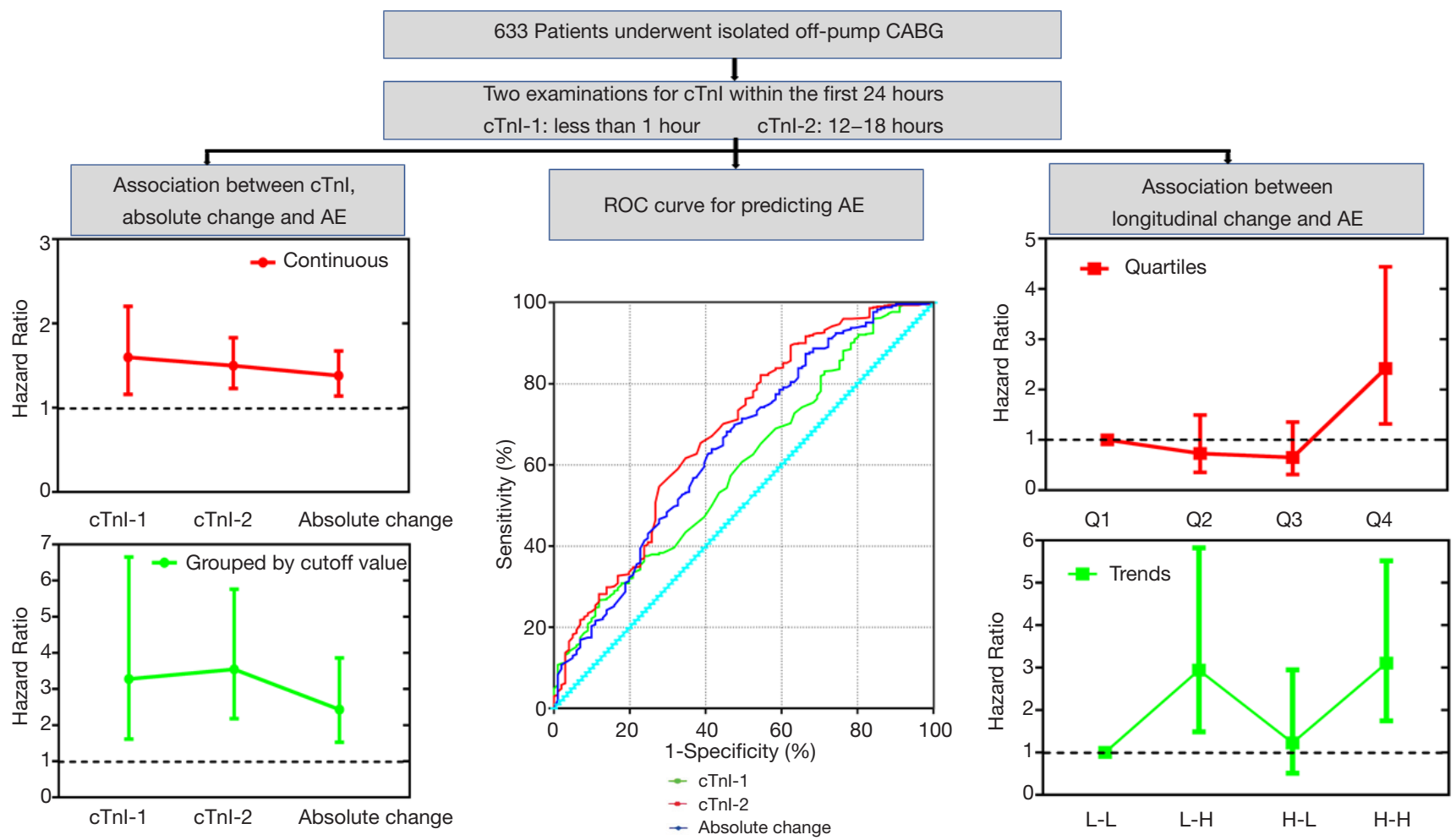

Figure 5 Central illustration of the association of early troponin I level and its longitudinal change with postoperative 30-day composite adverse events. Change in cTnI concentrations is expressed in quartiles: quartile $1<-0.0768 \mu \mathrm{g} / \mathrm{L},-0.0768 \mu \mathrm{g} / \mathrm{L} \leq \mathrm{quartile} 2 \leq 0.000 \mu \mathrm{g} / \mathrm{L}$, $0.000 \mu \mathrm{g} / \mathrm{L}<$ quartile $3 \leq 0.0908 \mu \mathrm{g} / \mathrm{L}$, quartile $4>0.0908 \mu \mathrm{g} / \mathrm{L}$, with quartile 1 as the reference. Change in cTnI concentrations is expressed in trends according to $\mathrm{c} T n \mathrm{I}$ concentrations at the first and second sampling times relative to the median of each time: $\mathrm{H}-\mathrm{H}$, high cTnI-1 and high cTnI-2; H-L, high cTnI-1 and low cTnI-2; L-H, low cTnI-1 and high cTnI-2; L-L, low cTnI-1 and low cTnI-2, with L-L as the reference. Absolute change in $\mathrm{c} T n \mathrm{nI}$ levels is defined as the absolute value of the first $\mathrm{c} T \mathrm{TnI}$ concentration minus the second $\mathrm{c} T \mathrm{n} \mathrm{I}$ concentration. The cutoff value was identified by the maximum Youden index value, and the group whose cTnI concentration was less than the cutoff value was used as the reference. CABG, coronary artery bypass grafting; AE, adverse events; ROC, receiver operating characteristic curve; cTnI-1, measurement of cardiac troponin I levels less than 1 hour after CABG; c'TnI-2, measurement of cardiac troponin I levels between 12-18 hours after coronary artery bypass grafting; cTnI-1, cardiac troponin I.

identify patients at an increased risk of complications. Our study demonstrated that a cTnI concentration at less than 1 hour after surgery was associated with composite adverse events, and this finding could potentially alert doctors to perform early therapeutic intervention to reduce patients' risk. Serial measurement of troponin is commonly used in patients with persistent myocardial injury, which is suspected to lead to poor prognosis. Our study quantitively showed that longitudinal change between cTnI levels from two time points within first 24 hours after CABG was significantly associated with poor clinical outcomes, and that patients with persistently high cTnI levels and those with progression from low to high $\mathrm{c} T \mathrm{nI}$ levels experienced a more than three-fold increased hazard of adverse events than patients who had persistently low cTnI concentrations.

\section{Limitations}

First, the single-center study design may have caused bias in the measurement of $\mathrm{c} T \mathrm{Tn}$, and postoperative cTnI levels were partly affected by surgeons' experience, the procedure duration, and other operative factors. Second, the study enrolled a relatively small number of patients who received off-pump CABG. Therefore, it would be valuable to confirm the association and replicate predictive capacity in an independently large cohort. 


\section{Conclusions}

There was a significant association between early c $T n I$ concentrations after CABG and adverse events even less than 1 hour after CABG. Longitudinal change in the $\mathrm{c} T \mathrm{TnI}$ level within 24 hours after CAGB is associated with the development of adverse events, and such data will be useful in identifying patients at an increased risk.

\section{Acknowledgments}

Funding: None.

\section{Footnote}

Reporting Checklist: The authors have completed the STARD reporting checklist. Available at http://dx.doi.org/10.21037/ jtd-20-1691

Data Sharing Statement: Available at http://dx.doi. org/10.21037/jtd-20-1691

Conflicts of Interest: All authors have completed the ICMJE uniform disclosure form (available at http://dx.doi. org/10.21037/jtd-20-1691). The authors have no conflicts of interest to declare.

Ethical Statement: The authors are accountable for all aspects of the work in ensuring that questions related to the accuracy or integrity of any part of the work are appropriately investigated and resolved. The study was conducted in accordance with the Declaration of Helsinki (as revised in 2013). The study was approved by institutional ethics board of Beijing Anzhen Hospital (No. ks2019018). All participants provided informed consent before taking part.

Open Access Statement: This is an Open Access article distributed in accordance with the Creative Commons Attribution-NonCommercial-NoDerivs 4.0 International License (CC BY-NC-ND 4.0), which permits the noncommercial replication and distribution of the article with the strict proviso that no changes or edits are made and the original work is properly cited (including links to both the formal publication through the relevant DOI and the license). See: https://creativecommons.org/licenses/by-nc-nd/4.0/.

\section{References}

1. Neumann FJ, Sousa-Uva M, Ahlsson A, et al. 2018 ESC/ EACTS Guidelines on myocardial revascularization. Eur Heart J 2019;40:87-165.

2. Thielmann M, Massoudy P, Jaeger BR, et al. Emergency re-revascularization with percutaneous coronary intervention, reoperation, or conservative treatment in patients with acute perioperative graft failure following coronary artery bypass surgery. Eur J Cardiothorac Surg 2006;30:117-25.

3. Domanski MJ, Mahaffey K, Hasselblad V, et al. Association of myocardial enzyme elevation and survival following coronary artery bypass graft surgery. JAMA 2011;305:585-91.

4. El Messaoudi S, Wouters CW, van Swieten HA, et al. Effect of dipyridamole on myocardial reperfusion injury: A double-blind randomized controlled trial in patients undergoing elective coronary artery bypass surgery. Clin Pharmacol Ther 2016;99:381-9.

5. Machado MN, Rodrigues FB, Grigolo IH, et al. Early Prognostic Value of High-Sensitivity Troponin T after Coronary Artery Bypass Grafting. Thorac Cardiovasc Surg 2019;67:467-74.

6. Holmvang L, Jurlander B, Rasmussen C, et al. Use of biochemical markers of infarction for diagnosing perioperative myocardial infarction and early graft occlusion after coronary artery bypass surgery. Chest 2002;121:103-11.

7. Gahl B, Gober V, Odutayo A, et al. Prognostic Value of Early Postoperative Troponin T in Patients Undergoing Coronary Artery Bypass Grafting. J Am Heart Assoc 2018;7:e07743.

8. Koppen E, Madsen E, Greiff G, et al. Perioperative Factors Associated With Changes in Troponin T During Coronary Artery Bypass Grafting. J Cardiothorac Vasc Anesth 2019;33:3309-19.

9. Fellahi JL, Gue X, Richomme X, et al. Short- and longterm prognostic value of postoperative cardiac troponin I concentration in patients undergoing coronary artery bypass grafting. Anesthesiology 2003;99:270-4.

10. Alexander JH, Smith PK. Coronary-Artery Bypass Grafting. N Engl J Med 2016;374:1954-64.

11. Lloyd-Jones D, Adams R, Carnethon M, et al. American Heart Association Statistics C and Stroke Statistics S. Heart disease and stroke statistics--2009 update: a report from the American Heart Association Statistics Committee 
and Stroke Statistics Subcommittee. Circulation 2009;119:480-6.

12. Kim LJ, Martinez EA, Faraday N, et al. Cardiac troponin I predicts short-term mortality in vascular surgery patients. Circulation 2002;106:2366-71.

13. Greenson N, Macoviak J, Krishnaswamy P, et al. Usefulness of cardiac troponin I in patients undergoing open heart surgery. Am Heart J 2001;141:447-55.

14. Thielmann M, Massoudy P, Schmermund A, et al. Diagnostic discrimination between graft-related and nongraft-related perioperative myocardial infarction with cardiac troponin I after coronary artery bypass surgery. Eur Heart J 2005;26:2440-7.

15. Thielmann M, Massoudy P, Neuhauser M, et al. Prognostic value of preoperative cardiac troponin I in patients undergoing emergency coronary artery bypass surgery with non-ST-elevation or ST-elevation acute coronary syndromes. Circulation 2006;114:I448-53.

16. Petäjä L, Rosjo H, Mildh L, et al. Predictive value of high-sensitivity troponin $\mathrm{T}$ in addition to EuroSCORE

Cite this article as: $\mathrm{Li} \mathrm{Y,} \mathrm{Li} \mathrm{Y,} \mathrm{Hu} \mathrm{Q,} \mathrm{Zheng} \mathrm{S,} \mathrm{Tian} \mathrm{B,} \mathrm{Meng}$ F, Chen Z, Han J, Wang S, Zhang H, Xu C, Jia Y, Jiao Y, Fu J, Meng X. Association of early elevated cardiac troponin I concentration and longitudinal change after off-pump coronary artery bypass grafting and adverse events: a prospective cohort study. J Thorac Dis 2020;12(11):6542-6551. doi: 10.21037/jtd-201691
II in cardiac surgery. Interact Cardiovasc Thorac Surg 2016;23:133-41.

17. Lehrke S, Steen H, Sievers HH, et al. Cardiac troponin $T$ for prediction of short- and long-term morbidity and mortality after elective open heart surgery. Clin Chem 2004;50:1560-7.

18. Mohammed AA, Agnihotri AK, van Kimmenade RR, et al. Prospective, comprehensive assessment of cardiac troponin T testing after coronary artery bypass graft surgery. Circulation 2009;120:843-50.

19. White HD. Pathobiology of troponin elevations: do elevations occur with myocardial ischemia as well as necrosis? J Am Coll Cardiol 2011;57:2406-8.

20. Thygesen K, Alpert JS, Jaffe AS, et al. Fourth Universal Definition of Myocardial Infarction (2018). J Am Coll Cardiol 2018;72:2231-64.

21. Muehlschlegel JD, Perry TE, Liu KY, et al. Troponin is superior to electrocardiogram and creatinine kinase $\mathrm{MB}$ for predicting clinically significant myocardial injury after coronary artery bypass grafting. Eur Heart J 2009;30:1574-83. 